\title{
Analysis on Vibration NVH of General Mechanical
}

\author{
Xuanming Zhao ${ }^{1, \text { a }}$
}

${ }^{1}$ College of Energy Engineering, Yulin University, Yulin City, Shaanxi Province, 719000

aemail,

\author{
Keywords: Analysis, Vibration NVH, General Mechanical
}

\begin{abstract}
With awareness of environmental protection is increasing, the government regulations on noise limits have become increasingly stringent, people's requirement for the comfort of general machinery is increasing. The NVH performance of general machinery will be the main trend of the future general machinery competition. With the rapid development of general machinery industry in China, the market competition of general machinery is increasingly fierce and the vibration noise of general mechanical has become a very important project to be evaluated.
\end{abstract}

\section{Introduction}

NVH improvement technology is reflected in the actual design of each system and each component of general mechanical. In the product development process, NVH is an essential item to consider in the development process. The basic idea is to first understand the mechanism of various vibration phenomena and clear the main exciting force system, conduction system and radiology systems. Then select the critical systems and key components associated with a variety of vibration, clear the $\mathrm{NVH}$ characteristics of these systems and components (vibration characteristics and acoustic characteristics). Of course, the overall performances of the vehicle are also related to the vibration characteristics, so we first need to understand the formation of the individual characteristics of the vehicle's overall performance and set a suitable target to the various characteristics. On this basis, given the various systems and components target value related to the desired design, so as to achieve the target through detailed design work. Finally, through the evaluation of each characteristic of various systems and components, we evaluate the integrated nature of the vehicle.

$\mathrm{NVH}$ phenomena are closely related to people's subjective feeling, when setting the target value, that is before the absolute evaluation of using vibration and noise values, etc., we should use the basic human feeling to make relatively subjective evaluation. And we should also compete and compare with the cars and the target vehicle, using the subjective evaluation, experience the level to design target reference value and evaluation value.

\section{The Definition of NVH}

$\mathrm{NVH}$ is the abbreviation of three English words noise, vibration and harshness. The specific explanation is as follows: Noise: the audible interference at frequencies between $20-5000 \mathrm{~Hz}$, the main feature is the evaluation frequency, level and quality. Vibration: the movement of the body that can be felt at frequency between 0.5 to $50 \mathrm{~Hz}$, wherein the main evaluations are frequency, level and direction. Harshness: the vibration and noise associated with the composite effect, rude, friction or discord feeling mainly related to the road incentives. Vibration and sound can have different effects on the driver and passenger. At the same time as the source of interference is to give people a positive quality movement, solid, smooth feel, and operation can be obtained feedback. NVH performance is an important performance to evaluate the vehicle, the customer is more concerned about the performance and it's an important evaluation aspect of commodity vehicle.

\section{The Regulatory Requirements Related to the NVH}

The national compulsory standards related to NVH performance is GB1495-2002- general machinery noise outside the vehicle acceleration with performance requirements. According to the 
current situation of the time, M1 class vehicle acceleration with the vehicle noise limit of 74 decibels. It should be noted, did not make provision for standard interior noise limits. Because the interior noise is an important aspect of the evaluation of vehicle comfort, if the interior noise exceeds a certain level, it will cause the user's complaints, will be punished by the markets, directly affect the efficiency of enterprises; therefore, even if there is no standard on the car within the noise limits as required under the respective general machinery manufacturing enterprises have been in the pursuit of a low noise level inside the car, designed to take a variety of methods.

\section{The Source of Vibration Noise and Transfer Path Analysis}

$\mathrm{NVH}$ phenomenon can be divided into such aspects according to operating conditions: vibration noise when starting, idling vibration noise, vibration noise during acceleration, constant speed cruising vibration noise and vibration noise when decelerating. It can be classified according to sound type: rumble, rip cracking sound, snapping sound, growl and whine like. According the sound source it can be divided into: power train noise, intake and exhaust noise, road noise, tire noise, high-speed wind noise, noise and other attachments.

Start the engine and run at idle speed, engine start power train noise vibration and noise: In cold start, since the oil viscosity is too high, the driving torque is too large, sometimes the valve actuator mechanism excessive noise, vibration and noise caused by the engine start work larger. Engine accessories operating noise, noise-generating engine accessory include: noise transmission chain, belt noise, pump noise, fuel pump noise, fan noise. Among them, the noise itself, as well as interior noise propagation through the various pipeline annex noise outside noise is airborne.

$\mathrm{H}$-throttle body vibration noise air or air conditioning at work: When empty H-throttle or air-conditioning operation, the engine will produce higher frequency excitation force, sometimes the natural frequency of the vehicle body and a wider range of systems coincide, causing the car more significant vibrator. For example: bending resonance of the exhaust system, the body resonance plate.

Vibration of start-up and acceleration of the noise conditions, the need to increase the engine output torque, the reaction force will cause the engine side of the pendulum vibrations that pass through the engine mounts to the vehicle body, the rise of the torque per unit time the larger the ratio, the stronger the feeling the impact of the occupant.

Idle start and accelerate the vehicle in addition to the above-described vibration phenomenon occurs, there are two vibration phenomenon is worth noting that due to the suspension spring-mass resonance and shaft angle changes induced vibrations.

Mechanism suspension spring resonance is as follows: Engine torque variation transmitted to the wheel through the shaft, drive shaft reaction force as an incentive to force, excite resonance of the body, this resonance phenomenon is accompanied by the roar of low-frequency vibration of the vehicle body resonance noise.

Constant velocity with noise and vibration of the vehicle body is the typical cyclical ups and downs of the road pavement. Undulating road surface by vehicle vibration, because the suspension stroke caused by excessive impact limit stopper issues, a considerable proportion of the number of problems over the past comfort, comfort is an important evaluation projects.

Use real vehicle test to verify the undulating road vehicle driving comfort. The test is usually performed on an appropriate undulating pavement road test field setting.

Undulation of the road is to simulate road conditions concrete repair roads. Acceleration measurement including crew cabin and seats three three-point acceleration measurement points a total of six points. The relationship between the maximum acceleration value of each measurement point and subjective evaluation scores were compared between. For a long time, it has been up and down the body, left and right, before and after the acceleration vector in three directions and evaluated. Similarly, the driver's seat also has the same effect, but the higher the driving seat acceleration measurement requirements, and measures much trouble, so generally ride comfort evaluation projects, mainly provided by the cab acceleration evaluated.

Paved road through a seam on the potholes, cracks, etc. or punch a single road, the body will 
produce excessive shock and vibration. The comfort is also an important indicator of evaluation. In theory, when the tire through the projection, the average length of contact of the tire and the road and the basic spatial frequency vibration components related to this case is the fundamental vibration shock and vibration up and down and back and forth direction. Road input vibration can stimulate the mass resonance, including resonance including tires, through suspension, the excitation vibration of the vehicle body.

\section{The NVH Test of General Machinery}

The mainly two indicators of NVH performance of general machinery are vibration and noise. In terms of vibration, the vibration of generic mechanical can cause early fatigue failure of certain components, thereby reducing the life of general machinery. In terms of noise, there are common external noises and mechanical noise within the general machinery, excessive noise within the general machinery both damage the driver's hearing, but also makes the driver fatigue quickly, and thus constitutes a very threat on the general machinery with safety. For consumers, their first concern is the size of the interior noise and vibration as well as the level of interior comfort; for the environment, mainly referring to vehicle emissions and noise emissions, it must meet the countries inside and outside the vehicle emissions and noise pollutant emission regulations. Any one of the new models in the vehicle development process must be tested to verify the performance indicators, and NVH performance for the same vehicle in the process of developing all aspects of the test must be verified, such as interior noise and vibration target validation test, the vehicle noise test target validation, engine mounting performance test verification. For $\mathrm{NVH}$ problems existing models exist can be performed by means of test analysis tests to find the cause of the problem, propose engineering feasibility of governance programs. Finally, experimental results verify governance. Thus we see the importance of experimental methods in the study of general mechanical $\mathrm{NVH}$ characteristics.

In the development process of new models, noise vibration indicators must first meet the mandatory regulations of the country or region. With the increasingly fierce market competition and customer demands for more comfort, NVH performance indicators have been forced to shift from the original regulations mandatory for customers. Therefore, in the actual development process, it is necessary to NVH performance of competing models tested, the benchmark value of the test; the second is to the development process models were NVH performance testing, benchmarking and comparative value. Through two testing process, submit the test results, conclusions or give a certain degree of subjective evaluation, to ensure to provide customers with "products" to meet customer requirements.

\section{The Noise Source Identification Technology of Troubleshooting}

The traditional method for noise measurement are mainly subjective evaluation method, respectively operate elimination, measurement of sound pressure, sound intensity measurement. With the development of acoustic measurement technology, quick and easy way to identify the source of the noise made the progress. The basic trend is the development of a new method of identifying the requirements of the test method for measuring the energy field, real-time analysis and processing of the measurement signal obtained, can improve the reliability of test results.

Subjective Evaluation Method. The human auditory system has a relatively sophisticated noise measurement system is more accurate identification ability to distinguish between different sounds, but also widely used in actual measurement of sound pressure or loudness and other indicators are based on the subjective feeling may be. Subjective identification method due to different people and different conclusions, in order to obtain high accuracy must be a lot of training to gain experience. In addition, subjective evaluation method is also difficult to make a quantitative assessment of the sound source, which is the main drawback.

Respectively Operate Elimination. When general machinery is working, there are hundreds of parts simultaneously and the early method to determine which part of the maximum radiated noise 
generally used is elimination. Elimination method is to first test measured objects (such as vehicles, engines, etc.) under certain conditions, the overall noise then it may be part of a larger issue of noise, or suspend its working methods to control the noise radiation. Again under the same conditions of work noise measurement test object, according to the principle of superposition of sound pressure level from the test results of the two noises can calculate the size of this part of the radiated noise. This method is simple, does not require sophisticated equipment and technology, but because of the overall vehicle system and its components are interrelated, a certain part of the demolition and stop work, it will affect other portions associated with them, so some of the data measured error is larger; in addition, this method time consuming, and there are some limitations, for example, on the surface of the radiation source of radiation where the most serious, this method cannot be concluded.

Lead Overlay. With stereotype made a similar machine with the portion of the surface of the cover, inside covered with sound-absorbing material such as glass fiber, in order to reduce reverberation, sound insulation inside the hood at least in I0dB above. When tested in the same conditions one by one to open the cover of a "window" of exposure each surface acoustic noise radiation source and the exposed surface at a distance measured sound pressure level at a distance, if the individual in order to open the "window" to measure, you can get on the main surface radiation area. The method commonly used in each engine surface radiated noise measurement.

Compared with the operation of respectively operate elimination, it cannot stop and impact due to some parts, as long as the cover tight, it is a more reliable method. Since the low-frequency noise isolation more difficult, it is only suitable for medium and high frequency noise source identification; moreover, the method used to spend a lot of time measuring and testing the acoustic environment have certain requirements (preferably in an anechoic chamber).

Sound Pressure Measurements. Sound pressure is a scalar, the sound pressure space at a point often influenced by the presence of the respective direction of the sound source, so from a point measured sound pressure difficult to determine where the main source of noise. However, under certain conditions, such as near-field measurement methods and through tube measuring method, when the effect of noise is suppressed in other directions, or can determine the main sources of noise from the measurement results of sound pressure. However, when using such a method to identify the main sources of noise, more complex operations, the efficiency is lower.

\section{Conclusion}

Through work and summary of the theory, we had a better understanding of tools of general mechanical vibration and noise inside the car and be applied in practice, and achieved certain results. According to the methods and experience explored in this article, we can comprehensively and systematically in-depth study the NVH performance of general machinery.

\section{References}

[1] Souhei Matsuyama, Shinichi Marayama,Booming Noise Analysis Method Based On Acoustic Excitation Test , SAE Paper 980588.

[2] ADAMS/Tire Option Version 11 MDI 1997

[3] Joe Juan Todd Depauw Vehicle Dynamics and NVH Tradeoff Studies Using ADAMS 1997 ADAMS

[4] Gupta V, Gunasekar T.J, Rao A, Kamarajan J and Summer S, Reverse Engineering Method for Developing Passenger Vehicle Finite Element Models, SAE Paper 1999-01-0083

[5] Jody R. Shaw, Blake K. Zuidema, New High Strength Steels Help Automakers Reach Future Goals for Safety, Affordability, Fuel Efficiency and Environmental Responsibility, SAE Paper 2001-01-3041

[6] Hiroyuki Kuwahara,Virtual Assessment Of Motorcycle Vibrations, Yoji Fukui-Honda R\&D CO.LTD-Japan 